\title{
75 Jahre Deutsche Gesellschaft für Lichtforschung
}

\section{E. G. Jung}

\author{
75 Years of the „Deutsche Gesellschaft für Lichtforschung“
}

\section{Zusammenfassung}

Zu Beginn des 20. Jahrhundert erlebte die Photobiologie einen ungeahnten Aufschwung durch die Heliotherapie einerseits und durch die Therapie der Tuberkulosen und die Rachitisprophylaxe mit künstlichen Lichtquellen anderseits. Die wissenschaftliche Durchdringung und die klinische Anwendung erforderten Kooperation der Fächer und benötigten gegenseitige Befruchtung. Dem wurde 1927 Rechnung getragen durch die Gründung der Deutschen Gesellschaft für Lichtforschung (DGfL) in Hamburg durch Prof. Hans Meyer, um Ärzte, Physiker und Meteorologen zusammenzuführen. Diese fächerübergreifende Konstruktion ist bis jetzt erhalten geblieben und prägt die DGfL maßgeblich. Gleichzeitig erfolge die enge Verknüpfung mit den internationalen Vereinigungen, die sich zunehmend intensivierte. Die Photobiologie hat dann gegen Mitte des Jahrhunderts, infolge therapeutischer Fortschritte und akzentuiert durch den 2. Weltkrieg, wesentlich an Bedeutung verloren. Erst neue Entwicklungen aus der Photochemie und der Biophysik rechtfertigten die Wiedererweckung resp. die Neugründung der DGfL im Jahre 1953. Seit den internationalen Photobiologenkongressen 1968 in New Hampshire/USA und in Bochum 1972 zeichnen sich neue und gewichtige klinische Schwerpunkte ab, welche die Medizin (Dermatologie und Endokrinologie), die Epidemiologie und die Gerätetechnik eng mit der zellulären und molekularen Biologie verbanden. Zudem eröffnet die Photoimmunologie seit gut 10 Jahren ganz neue Felder, welche breite interdisziplinäre Kooperationen erfordern und die DGfL neuen Zielen entgegenführen.

\section{Abstract}

Photobiology made a tremendous progress at the beginning of the $20^{\text {th }}$ century. This was due to heliotherapy as well as to the phototherapy of tuberculosis and the prevention of rickets by artificial light sources. The scientific intermingling and the clinical application need cooperation of the sciences and mutual inspiration. Therefore the „Deutsche Gesellschaft für Lichtforschung“ (DGfL) was founded 1927 in Hamburg by Prof. Hans Meyer, to bring together Medicine, Meteorology and Physics. The interdisciplinarity is one of the chracteristics of the DGfL and it is made to last. In the same time many connections with international associations are built up and installed to endure. Photobiology lost considerably in importance up to the middle of the century by reasons of therapeutical progress as well as by World War II. After this devasting period, new developments in photochemistry and in biophysics finally justified the reestablishment of the DGfL in 1953. The international congresses of photobiology, 1968 in New Hampshire/USA and in Bochum 1972 were characterized by new and important highlights, bringing together Medicine (Dermatology and Endocrinology), Epidemiology and Technology with the cellular and molecular Biology. In addition, Photoimmunology is in progess, since more than 10 years. These novel fields of photobiology, demanding even more interdisciplinary cooperation, will open very new horizons to the DGfL. 
ASP American Society for Photobiology

(neu: Photomedicine Society)

C.I.L. Comité International de la Lumière

C.I.P. Comité International de Photobiologie

DDG Deutschsprachige Dermatologische Gesellschaft

DGfL Deutsche Gesellschaft für Lichtforschung

ESP European Society of Photobiology

ESPD European Society for Photodermatology

LiTG Lichttechnische Gesellschaft

\section{Einleitung}

Das ausgehende 19. und der Beginn des 20. Jahrhunderts zeichnen sich nicht nur durch die Naturbewegung, „Jugendstil“, Körperkultur und Sport aus [1], sondern auch durch die Verbreitung und Anerkennung der Heliotherapie (Lichttherapie).

Schon 1855 errichtete Arnold Rikli (1823-1906) im damaligen Völdes im österreichischen Oberkrain (jetzt Bled in Slowenien) ein Sanatorium zur kombinierten Sonnen- und Dampfbad-Therapie [2]. Die Heliotherapie wurde unterstützend bei chronischen Krankheiten und zur Therapie von Hauterkrankungen, Infektionen und schlecht heilenden Wunden sowie zur Prophylaxe und Behandlung der Rachitis eingesetzt. Die Behandlung mit der natürlichen Globalstrahlung, die im Gebirge und an der Küste einen größeren ultravioletten Anteil enthält und weniger durch klimatische Imponderabilien beeinträchtigt ist, setzt sich immer mehr durch. Aus diesem Grund wurde die Heliotherapie als Teil der Klimabehandlung besonders im Hochgebirge von Samaden (O. Bernhard) und Leysin (A. Rollier), im Mittelgebirge und auf den küstennahen Inseln Norderney, Sylt und Rügen eingesetzt. Die besten Effekte der Heliotherapie wurden neben der Rachitis wohl bei der Knochen- und Lungentuberkulose erreicht $[1,3]$.

Mochte dies zunächst als Modeströmung gelten, folgte bald die aus medizinischen Erfolgen genährte Überzeugung der vorbeugenden und therapeutischen Wirkung. So bot die Heliotherapie Anreiz genug für neue und vielfältige Forschungsaufgaben. $\mathrm{Zu}$ dem war dies auch „die hohe Zeit der Ernte“ in der klinischen Dermatologie mit der Beschreibung und Erforschung der lichtabhängigen Hautkrankheiten und der Lichtdermatosen [1,3,4].

\section{Voraussetzungen zur Grüindung der DGfL}

Im Jahre 1896 beobachtete der dänische Arzt Niels Ryberg Finsen (1860 - 1904), dass die Hauttuberkulose seiner Katze sich unter der Sonnenbestrahlung besserte. Er entwickelte daraus die Lichttherapie der Hauttuberkulose, nachdem er zusammen mit seinem Schwager und Nachfolger Axel Reyn (1872 - 1935) mit der „Finsen-Reyn-Lampe“, einer gefilterten, UV-reichen Kohlenbogenlampe, die Voraussetzung für die lokale und dosierbare Bestrahlungsmöglichkeit schuf. Die Kontaktbestrahlung der Hauttuberkulose war mit einer Heilungsquote von über $90 \%$ außerordentlich erfolgreich. Finsen erhielt dafür 1903 den Nobelpreis für Medizin und Physiologie und gründete in Kopenhagen ein eigenes Institut für dermatologische Lichttherapie. Die Kontaktbe- strahlung der Hauttuberkulose fand weite Verbreitung, Anerkennung und Ergänzung. So entwickelte der Dermatologe Ernst Kromayer (1862 - 1933) 1906 in Berlin unter Verwendung des 1905 von Küch entdeckten Quarzglas-Verfahrens eine wassergekühlte Quecksilber-Hochdrucklampe zur Kontaktbestrahlung der Hauttuberkulose, welche in der Folge weitgehend die von Svend Lomholt weiterentwickelte Finsen-Reyn-Lampe verdrängte. Die Quarzlampen-Gesellschaft wurde gegründet, die (jetzt in Hanau) eine Vielzahl von Quecksilberdampf-Hochdrucklampen entwickelte für klinische und experimentelle Belange. Dazu gehört auch die weitverbreitete „Höhensonne“ als eingetragenes Warenzeichen der Original-Hanau-Quarzlampengesellschaft (Abb.1). Ergänzt durch Entwicklungen der Osram GmbH München, Berlin und der Deutschen Philips GmbH, Hamburg, standen nun Strahlenquellen und Messeinrichtungen zur Verfügung, um die Lichtbiologie der Haut und deren Pathologie exakt zu erforschen. Physiker und Meteorologen kümmern sich um Globalstrahlung und Strahlungsklima, die Photochemie nimmt ihren Anfang und die Dermatologen bemühen sich zusammen mit Strahlenphysikern um die akuten (Erythem) und chronischen Lichteffekte an der Haut, unter Einschluss der Lichtkarzinogenese. Das Erythem, sein Verlauf, Aktionsspektren und Wirksamkeitskurven, die minimale Erythemdosis (MED) und der Lichtschutzfaktor (LSF) werden eingeführt und charakterisiert [1,4].

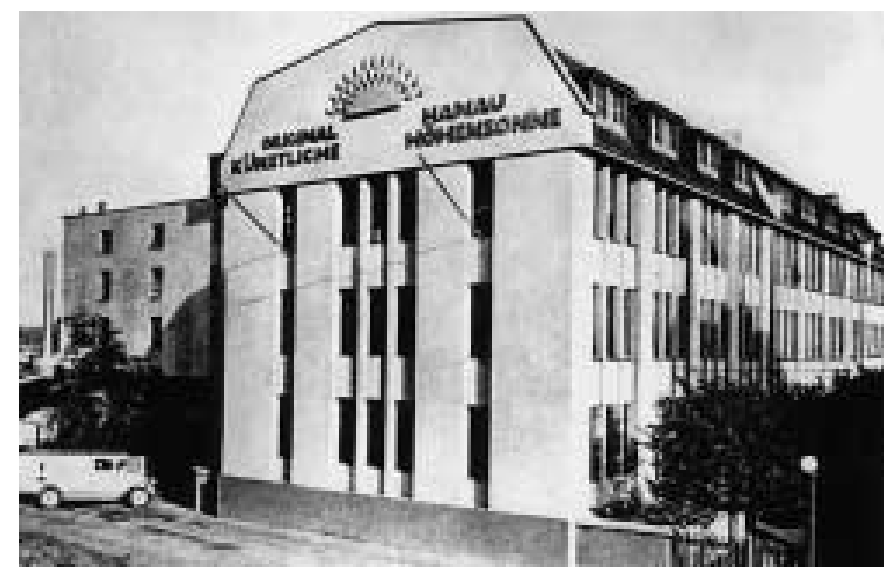

Abb. 1 Die Quarzlampengesellschaft in Hanau 1927, dem Gründungsjahr der DGfL (Werkfoto).

Lichtbiologie, Dermatologie, Physik und Chemie, aber auch Meteorologie und Klimatologie, bilden die Partner einer konzentrischen, multidisziplinären Bemühung, die strukturiert, koordiniert und stimuliert zu werden verdient. Dazu erfolgte die Gründung der „Deutschen Gesellschaft für Lichtforschung und Heliotherapie“" 1927

Dies erfolgte in Hamburg auf Anregung des Direktors des Eppendorfer Krankenhauses, Prof. Dr. L. Brauer, „mit dem Ziele, alle auf die Erforschung des Strahlungsklimas, der Lichtbiologie und der Lichttherapie gerichteten Bestrebungen zusammenzufassen und zu fördern, und Ärzte, Physiker und Meteorologen zu gemeinsamer Arbeit zusammenzuführen“ [5,6]. Zum Vorsitzender der Gesellschaft wurde Prof. Dr. Hans Meyer (1877-1964) gewählt (Abb. 2), der einen bemerkenswerten Werdegang hat. Nach der 


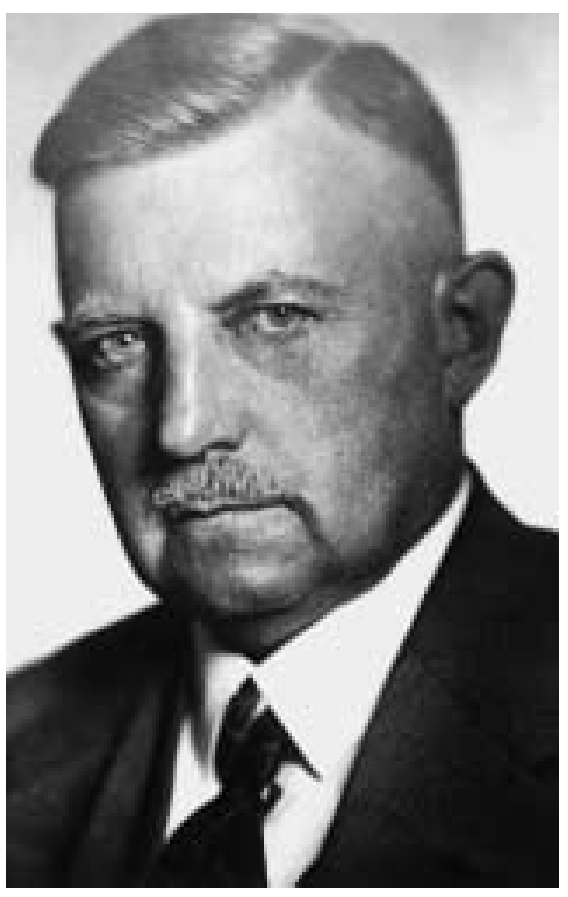

Abb. 2 Prof. Hans Meyer, Bremen, 1877-1964, Gründungspräsident der DGfL 1927-1930 (Bild: Krankenhaus Sankt Jürgen Straße, Bremen).

Assistentenzeit in der Dermatologie Kiel (E. v. Düring) wechselte er in die Physiologische Chemie nach Straßburg (F. Hofmeister), in die innere Medizin nach Basel (D. Gerhardt) um wieder in die Dermatologie zu gehen, zunächst nach Paris (L. Brocq und R. Sabouraud) und dann nach Bern (J. Jadassohn). Und nochmals wechselnd, ging er nach München zu H. Rieder, wieder in die Radiologie. Zurück in Kiel, habilitierte er sich 1911 „zwischen den Fächern“ für „Röntgenkunde und Lichttherapie“ und gründete ein "Strahlenforschungsinstitut“ an der Dermatologie (V. Klingmüller). Im Jahr 1920 lehnte er den Ruf auf das Ordinariat der Dermatologie in Greifswald ab, um 1921-1936 als Direktor die neu gegründete Röntgen- und Lichtabteilung an den Krankenanstalten seiner Heimatstadt Bremen, der heutigen „Professor Hans Meyer Klinik“ (Abb. 3) zu übernehmen. Nach dem Krieg leitete er 1946 - 1950 ad interim die Universitätsstrahlenklinik in Marburg [7-10].

Der Gründungskongress der DGfL findet am 27. und 28. Mai 1927 in Hamburg statt. Die erste Tagung wird ebenfalls in Hamburg, am 20.9.1928 abgehalten [11], und zwar im Rahmen der 90. Versammlung der Gesellschaft Deutscher Naturforscher und Ärzte, als deren Sektion 23. Themen waren Strahlenphysik, Biologie und Lichttherapie.

Die im Jahr 1912 vom Radiologen Hans Meyer, Bremen, gegründete Zeitschrift „Strahlentherapie“, offizielles Organ der Deutschen Röntgengesellschaft, wird ab 1928 auch zum offiziellen Organ der DGfL [11].

Die nächste Tagung findet am 25./26.9.1929 gemeinsam mit der Deutschen Pharmakologischen Gesellschaft in Münster statt. Schwerpunkt-Themen sind Vitamin D, Rachitis, Therapie der Tuberkulose und Großstadt-Luftverschmutzung [12]. Eine weitere Tagung fand vom 5./6.9.1930 in Dresden gemeinsam mit der Deutschen Gesellschaft für Hygiene statt und beschäftigte sich mit neuen Strahlungsquellen, Strahlungsmessungen und erneut

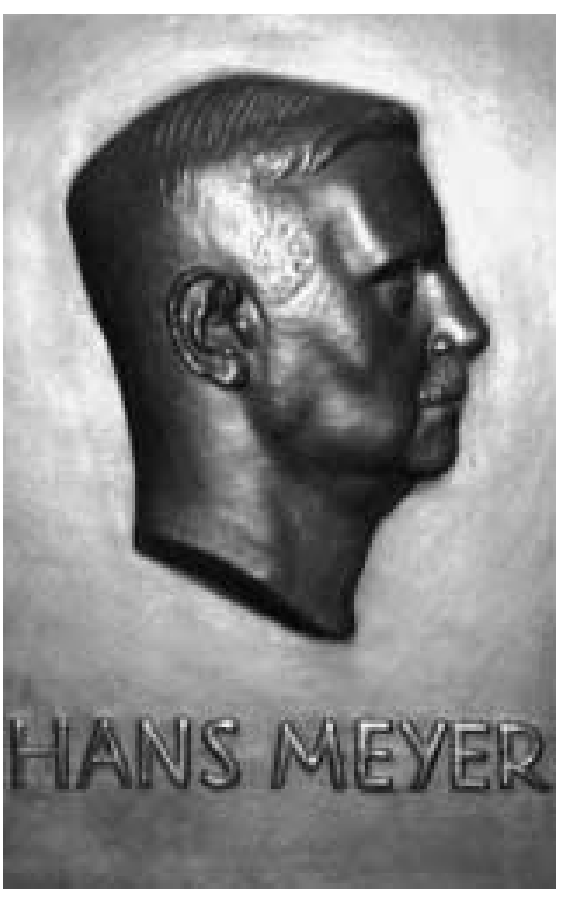

Abb. 3 Halbrelief von Hans Meyer in Bronze in der Eingangshalle der „Prof. Hans Meyer Klinik“" in Bremen (Foto: Prof. F. Bahmer, Bremen).

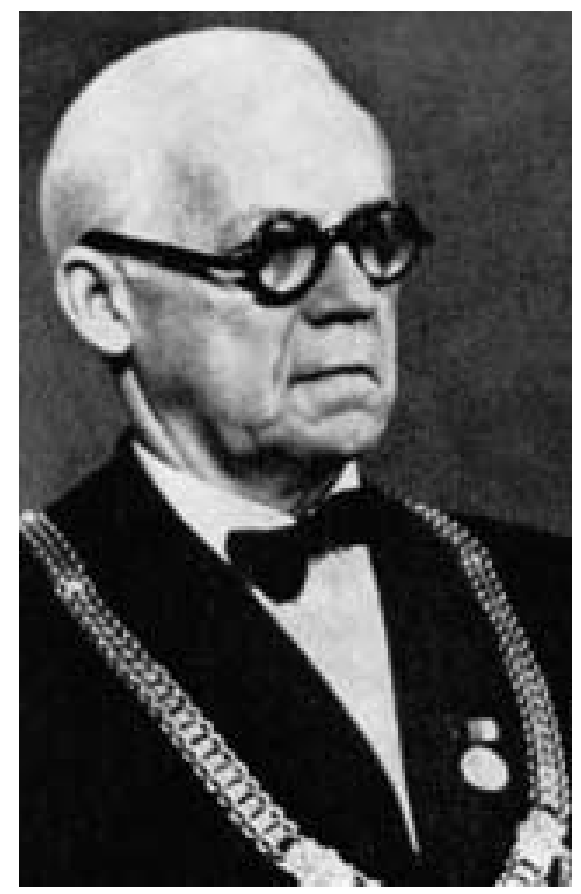

Abb. 4 Prof. Walter Friedrich, Berlin, 1883-1968, Präsident der DGfL $1930-1953$ (Bild aus [15]).

mit der Rachitis [13]. Der Vorsitz der DGfL ging an Prof. Walter Friedrich, Berlin (Abb. 4) über und Dr. Hans Schreiber, auch aus Berlin, wurde als Sekretär gewählt. Prof. Walter Friedrich [14] studierte Physik in Genf und München, promovierte bei W. Röntgen in München und erfuhr seine Formation als Strahlenphysiker bei A. Sommerfeld und M. v. Laue ebendort. Für die Jahre 1914-1922 etablierte er als Abteilungsleiter der UniversitätsFrauenklinik Freiburg (B. Krönig) die dortige Strahlentherapie. Die Berufung nach Berlin erfolgte 1922, wo er von 1923-1959 Direktor des Instituts für medizinische Physik an der Universität Berlin und auch Rektor der Humboldt-Universität zu Berlin 1949 - 1951 war. Zudem war er von 1947 - 1959 Direktor des Institutes für Medizin und Biologie der Deutschen Akademie der 


\begin{tabular}{|c|c|}
\hline \multicolumn{2}{|c|}{ Deutsche Gesellschaft für Lichtforschung (DGfL) } \\
\hline \multicolumn{2}{|l|}{ Die Vorsitzenden: } \\
\hline $\begin{array}{l}\text { Hans Meyer, Bremen und Marburg } \\
30.7 .1877-11.4 .1964\end{array}$ & $\begin{array}{l}\text { Gründungsvorsitzender } 1927 \\
\text { Ehrenpräsident } 1959\end{array}$ \\
\hline $\begin{array}{l}\text { Walter Friedrich, Berlin } \\
25.12 .1883-16.10 .1968\end{array}$ & $1930-1953$ \\
\hline $\begin{array}{l}\text { Boris Rajewsky, Frankfurt a. M. } \\
\text { 19.7.1893-22.11.1974 }\end{array}$ & $\begin{array}{l}1953-1965 \\
\text { Ehrenvorsitzender } 1965\end{array}$ \\
\hline $\begin{array}{l}\text { Rudolf Schulze, Hamburg } \\
13.5 .1906-16.7 .1974\end{array}$ & $1965-1974$ \\
\hline $\begin{array}{l}\text { Günther O. Schenk, Mülheim/Ruhr } \\
\text { 14.5.1913-25.3.2003 }\end{array}$ & $\begin{array}{l}\text { 1974-1978 } \\
\text { Ehrenmitglied } 1993\end{array}$ \\
\hline $\begin{array}{l}\text { Arthur Wiskemann, Hamburg } \\
\text { geb. 28.4.1922 }\end{array}$ & $\begin{array}{l}\text { 1978-1985 } \\
\text { Ehrenmitglied } 1993\end{array}$ \\
\hline $\begin{array}{l}\text { Ernst G. Jung, Mannheim } \\
\text { geb. 3. 3.1932 }\end{array}$ & $\begin{array}{l}1985-1995 \\
\text { Ehrenmitglied } 2000\end{array}$ \\
\hline $\begin{array}{l}\text { Erhard Hölzle, Oldenburg } \\
\text { geb. 14.5.1947 }\end{array}$ & ab 1995 \\
\hline \multicolumn{2}{|l|}{ Die Sekretäre: } \\
\hline $\begin{array}{l}\text { Hans Schreiber, Berlin } \\
\text { geb. } 1902\end{array}$ & $1928-1951$ \\
\hline Heinrich Pfleiderer, Kielu. Westerland & $1951-1959$ \\
\hline $\begin{array}{l}\text { Ernst O. Seitz, Hanau } \\
22.5 .1901-14.2 .1973\end{array}$ & $1959-1968$ \\
\hline $\begin{array}{l}\text { Arthur Wiskemann, Hamburg } \\
\text { geb. 28.4.1922 }\end{array}$ & $1968-1976$ \\
\hline $\begin{array}{l}\text { Volker Schaefer, Hanau } \\
\text { geb. 29.2.1932 }\end{array}$ & $1976-1995$ \\
\hline $\begin{array}{l}\text { Jan Krutmann, Düsseldorf } \\
\text { geb. 3.4.1959 }\end{array}$ & ab 1995 \\
\hline \multicolumn{2}{|l|}{ Die Reihe der Kassenwarte: } \\
\hline A. Schraub, Frankfurt a. M. & $1951-1959$ \\
\hline Horst Bücker, Frankfurt a. M. & $1959-1972$ \\
\hline Horst-Dieter Mennigmann, Frankfurt a. M. & $1972-1990$ \\
\hline Peter Bocionek, Stuttgart & $1900-1999$ \\
\hline Gerhard Saalmann, Herford & ab 1999 \\
\hline
\end{tabular}

Abb. 5 DGfL, die Vorsitzenden, Sekretäre und Kassenführer.

Wissenschaften zu Berlin und Präsident dieser Akademie 1951 - 1956 [15]. Seine Heimatgemeinde Aschersleben ehrte ihn mit einem Kirchenfenster.

Weiter ging es mit den Tagungen der DGfL im jährlichem Rhythmus, so auch in Bremen zusammen mit der Deutschen Röntgengesellschaft und zusammen mit der Deutschen Dermatologischen Gesellschaft (DDG) 1939 in Breslau zum Thema „Strahlung, Klima und Haut“.

So hat die Lichtforschung aus der klinischen Dermatologie heraus und dank der erfolgreichen Lichttherapie der Hauttuberkulose empirisch enorme Anstöße erfahren, der von der Industrie mit einem innovativen Schub für neue, bessere, gezielter anwendbare und sichere Bestrahlungsgeräte beantwortet wurde. Die Lichtbiologie begleitete experimentell und legte die messbaren und quantifizierbaren Grundlagen sowohl der sofortigen Lichtwirkung auf die Haut (Erythem: Wirksamkeitskurve, Gradation, minimale Erythemschwelle, Pigmentierung etc.) als auch der Späteffekte, wie Lichtschwiele, Pigmentstörungen und Lichtkarzinogenese. Dem Zusammenwirken der Grundlagen aus
Physik und Chemie mit der Geräteentwicklung, der Biologie und der therapeutischen Anwendung hat sich die DGfL ganz wesentlich und erfolgreich angenommen. Immer wieder wurden die neu entwickelten Geräte auf ihre klinische Verwendungsfähigkeit geprüft und die Indikationen und Grenzen abgesteckt. Folgerichtig kamen diese Bemühungen mit fortschreitendem Erfolg immer näher und intensiver in den Bereich der klinischen Dermatologie. Dem wurde auch in der Thematik der Tagungen Rechnung getragen.

Mit Fug und Recht bezeichnet man diese Zeitspanne als einen ersten Höhepunkt der experimentellen und klinischen Lichtforschung. Er fand sein abruptes Ende 1939 durch den Zweiten Weltkrieg.

\section{„Überwinterung“ während des Krieges 1939-1945}

In den Jahren des Krieges 1939-1945 und den ersten Nachkriegsjahren fand gleichsam eine „Überwinterung“ der DGfL in der Schweiz statt. Dies erfolgte in der Dermatologie durch Guido Miescher (1887 - 1961), Walter Burckhardt (1905-1971), beide in Zürich sowie in der Heliotherapie durch A. Rollier (1874-1954) in Leysin, O. Bernhard (1861-1939) im Engadin und in den Davoser Tuberkulosen-Sanatorien. Zudem besteht seit 1907 in Davos das von Carl Wilhelm Max Dorno (1865 - 1942) gegründete „Meterologisch-Physikalische Observatorium Davos" [16] mit Außenstellen im Engadin auf Muottas Muraigl (2450 m.ü.M.) und im Wallis auf dem Gornergrat (3126 m. ü. M.). Hier wurde mit besonderer Intensität die Globalstrahlung im Hochgebirge erfasst (T. Bener 1960). Zusammen mit dem Hamburger Strahlenphysiker Rudolf Schulze (1906-1974) und dem von Wien nach Philadelphia/USA emigrierten Dermatologen Fred Urbach haben sie sich erfolgreich bemüht, die Tagesund Monatsschwankungen zu Jahreskurven zusammenzufügen und regionale Besonderheiten sowie langfristige Schwankungen zu eruieren. Dies stellt die Basis dar für die Messungen und Berechnungen zum Einfluss der UV-bedingten Ozon-Veränderungen in der Stratosphäre.

\section{Internationale Bemühungen}

Ausgehend von der Feststellung, dass auf den internationalen Kongressen die Bedeutung des Lichtes allzu oft nicht genügend gewürdigt werde, ist auf Initiative von Dr. A. Rosselet, Professor an der Medizinischen Fakultät in Lausanne, eine „Erste Internationale Lichtkonferenz" einberufen worden. Diese fand am 11.9.1928 mit den zwei Abschnitten „Physikalische Grundlagen“ und „Biologie“ in Lausanne statt, während der Abschnitt über die Therapie zu Ehren von Dr. A. Rollier nach Leysin verlegt wurde $[17,18]$. Es versammelten sich unter dem Vorsitz von A. Rosselet 30 Wissenschaftler aus 8 Ländern, darunter J. Meier, J. Saidman, H. Jausion, Dr. Roussel (F), A. Rosselet, A. Rollier, E. Bach, L. Exchaquet (CH), R. Ledent (B), M. Lewick (UK), W. Friedrich, H. Schreiber, Dr. Peemöller (D), Axel Reyn, Prof. Sonne (DK), Prof. Ceresole (I) und E. Mayer (USA).

Zusätzlich zur fachübergreifenden Diskussion wurden zwei richtungweisende Beschlüsse gefasst: 
Die Gründung einer Vereinigung zur internationalen Kooperation in allen Belangen der Photobiologie unter dem Namen „Comité International de la Lumière“ (C.I.L.). Dieses vereinigt alle nationalen Gruppen oder Komitees engagierter Lichtforscher. Axel Reyn (Kopenhagen) wurde zum ersten Präsidenten des C.I.L., A. Rosselet (Lausanne) zum Sekretär gewählt. Das deutsche Lichtkomitee wurde von der DGfL eingesetzt.

Das C.I.L. wurde beauftragt, folgende Forschungsfelder zu stimulieren [17]:

- Physik und Chemie des Lichtes unter Einschluss der Messmethoden,

- Biologie und Pathologie der Lichtwirkung,

- Licht zur Therapie und Gesunderhaltung.

Dazu sollen in 4-jährigen Abständen „Internationale Lichtkonferenzen" abgehalten werden mit wechselnden Standorten. Zusätzlich sind internationale Tagungen zu speziellen Schwerpunkten vorgesehen. Für spezielle Fragestellungen können themenorientierte Unterkomitees einberufen werden.

Drei Internationale Lichtkonferenzen wurden veranstaltet:

- Paris 1929 unter dem Vorsitz von J. Saidman, Paris, mit Teilnehmern aus 8 Ländern.

- Kopenhagen 1932 unter dem Vorsitz von Axel Reyn, Kopenhagen, mit 42 Teilnehmern aus 12 Ländern. Der Festvortrag „Light and Life“ wurde vom Dänischen Nobelpreisträger Niels Bohr gehalten.

- Wiesbaden 1936 unter dem Vorsitz von Walter Friedrich, Berlin, mit 317 Teilnehmern aus 27 Ländern [19].

Nach dem Zweiten Weltkrieg wurde das C.I.L. 1950 von W. Mörikofer $(\mathrm{CH})$, R. Latarjet $(\mathrm{F})$ und A. Hollaender (USA) wiedererweckt. Formalisiert wurde dies anlässlich der „Journées Internationales de la Lumiére" in Paris vom 26.-30.9.1951. Das C.I.L. wurde in „Comité International de Photobiologie“ (CIP) umbenannt. Neuer Präsident wurde W. Mörikofer (Davos, $\mathrm{CH}$ ) und Jean Meyer (Paris) sein Sekretär. Erstmals waren auch wieder offizielle Vertreter aus Deutschland dabei, unter der Führung von Walter Friedrich, Berlin. Das CIP wurde beauftragt, ab 1954 (Amsterdam) den „International Congress of Photobiology“ in 4-jährigen Abständen zu veranstalten. Diese multidisziplinären Kongresse fanden bisher unter Einbezug von Europa, Asien und Amerika schon 13-mal mit großem Erfolg und enormen Beteiligungen statt.

Von besonderer Bedeutung war der Kongress 1968 in New Hampshire/USA mit 755 Teilnehmern aus 34 Ländern. George Porter (UK) hielt den Hauptvortrag über Flash-Photolyse und schnelle photochemische Reaktionen, wofür er 1967 den Nobelpreis für Chemie erhalten hatte. Zudem zeigte James Cleaver (UK) erstmals, dass den Lichttumoren der seltenen Erbkrankheit Xeroderma pigmentosum ein Defekt der Nucleotid Excision Reparatur (NER) zugrunde liegt.

Das CIP verwaltet auch den Fonds der Finsen-Stiftung in Erinnerung an den dänischen Arzt und Lichtforscher Niels Ryberg Finsen (1860 - 1904), der 1903 den Nobelpreis für Medizin und Physiologie für seine bahnbrechenden Leistungen zur Phototherapie der Tuberkulose erhielt. Im Jahre 1937 wurde erstmals die Finsen-Goldmedaille für herausragende Verdienste in der Photobiologie (an C. Dorno, Davos/CH) vergeben, die seit 1951 jeweils anlässlich der internationalen Kongresse für Photobiologie feierlich verliehen wird [17].

\section{Neugrüindung der DGfL 1953}

Nach dem Krieg dauerte es bis ins Jahr 1951, als sich die Reste der DGfL, erneut unter der Führung von Hans Meyer, jetzt Marburg, zu einer Tagung am 2.5.1951 in Baden-Baden trafen und zusammen mit den südwestdeutschen Tuberkuloseärzten und dem deutschen Lichtkomitee der C.I.L. tagten [20]. Die Weiterführung der DGfL, resp. eine Wiedererweckung wurde beschlossen. Dies erfolgte 1953 durch eine neue Generation interessierter Lichtforscher, die sich in Bad Homburg trafen und die DGfL neu gründeten, resp. wieder formierten. Zum ersten Vorsitzenden wurde der Frankfurter Biophysiker Prof. Dr. Dr. Boris Rajewsky (1893 - 1974) gewählt (Abb. 6). Dieser war als Schüler und Nachfolger von Fr. Dessauer von 1937-1966 Direktor des Max-Planck-Instituts für Biophysik in Frankfurt a. M. [21,22].

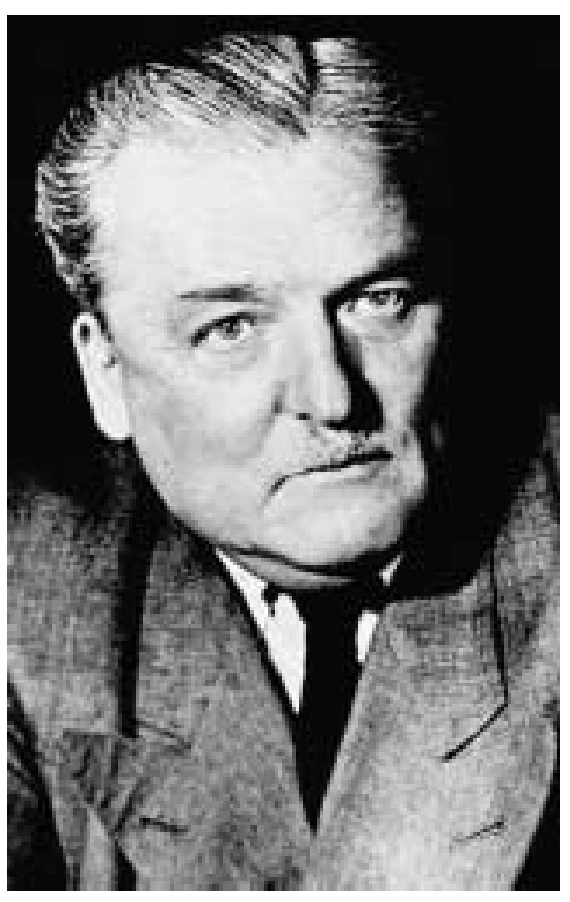

Abb. 6 Prof. Boris Rajewsky, Frankfurt a. M., $1893-1974$, Vorsitzender der DGfL 1953-1965 (Foto: Archiv der MaxPlanck-Gesellschaft Berlin).

Am 2.8.1955 wird die Deutsche Gesellschaft für Lichtforschung e.V. (DGfL) als Verein konstituiert und beim Amtsgericht Frankfurt eingetragen. Sie befasst sich satzungsgemäß mit der „Erforschung der Wirkung der optischen (ultravioletten, sichtbaren und infraroten) Strahlung auf die lebende Materie und Organismen, sowie mit der Förderung ihrer Anwendung in der Heilkunde. Der Verwirklichung dieser Ziele dient die enge Zusammenarbeit ihrer Mitglieder in Forschung und Lehre, auf wissenschaftlichen Tagungen und in Sonderkommissionen. Diese Formulierung ist bis heute in der Satzung der DGfL beibehalten worden (Amtsgericht Frankfurt/Main, Vereinsregister VR 4886). 
Schon bei den ersten Treffen wurde klar, dass nicht mehr an den Höhepunkt der Lichtforschung anzuknüpfen war, der 1939 durch den Krieg abgebrochen wurde. Die Behandlung der Tuberkulose, deren Häufigkeit und Bedeutung zwar zugenommen hatte, wurde durch die Entwicklung moderner oraler Tuberkulostatika dominiert. Dadurch wurde auch die Kontaktbestrahlung der Hauttuberkulose endgültig abgelöst. Zur Motivation der Gerätehersteller, denen es nach dem Krieg auch an Kraft fehlte, mangelte es an neuen Impulsen aus der Klinik. Andere Bedürfnisse standen im Vordergrund. Dies zeigte sich schon bei der ersten Tagung mit der DDG 1953 in Frankfurt/Main mit dem gemeinsamen Thema „Lichtdermatosen und Lichtschutz“. Die nachfolgenden Tagungen 1959 in Bad Nauheim [20], 1961 in Hanau und 1962 in Frankfurt/Main dienten der Bestandsaufnahme und Zielsuche im Bereich der Lichtbiologie, der Mikrobiologie und der Gerätetechnik. Tagungen in Westerland/Sylt 1962 und am Timmendorfer Strand 1965 hatten das Strahlungsklima zum Thema und die Lichtwirkung auf Pflanzen, Tiere und natürlich auf den Menschen insgesamt und speziell auf dessen Haut.

1965 wurde Herr Prof. Rudolf Schulze (1906 - 1974) zum ersten Vorsitzenden der DGfL gewählt (Abb. 7). Er stammte als Strahlenphysiker aus dem Berliner Institut von Prof. W. Friedrich und war 1952 - 1971 Direktor des Meteorologischen Observatoriums Hamburg des Deutschen Wetterdienstes [23]. Die Aufgabe des Sekretärs wurde von 1959-1968 von Prof. E. O. Seitz, Hanau (Abb. 8), geführt und anschließend von Prof. A. Wiskemann, Hamburg, 1968 - 1976. Die Kasse wurde von Prof. Horst Brücker 1959- 1972 verwaltet.

Die nächsten Tagungen waren mit den Dermatologen zusammen. Sie beschäftigen sich 1963 in Zürich und 1968 in Tübingen im Rahmen der DDG-Tagungen, sowie 1966 in Berlin und 1970 in Hamburg jeweils mit den regionalen Dermatologischen Gesellschaften vorwiegend mit Biologie und Diagnostik von Lichtdermatosen, als auch mit der Phototherapie in neuen Varianten.

Ein erster und bemerkenswerter Höhepunkt war der „6. International Congress of Photobiology" vom 21.-25.8.1972 in Bochum mit 650 Teilnehmern aus 32 Ländern. Ausgerichtet wurde er vom C.I.P. zusammen mit der DGfL durch ein nationales Lichtkomitee unter dem Vorsitz von Günther O. Schenck, Mülheim/Ruhr und dessen Sekretär Hagen Tronnier, Dortmund. Alle Themenkreise wurden abgehandelt, wobei die internationalen Schwerpunkte und Defizite mit denjenigen auf nationaler Ebene weitgehend übereinstimmten, wenn auch ein angloamerikanischer Vorsprung im molekularbiologischen Bereich deutlich wurde. Von besonderer Tragweite erwiesen sich die experimentellen Versuche zur und die ersten klinischen Erfolge mit der Photochemotherapie (Psoralen als Sensibilisator mit nachfolgender UVA-Bestrahlung, PUVA) bei der Psoriasis [24].

Bemerkenswerterweise wurde die „American Society for Photobiology“ (ASP) damals in Bochum gegründet.

Im Jahr 1974 übernahm der 1913 geborene Strahlenchemiker Prof. Günther O. Schenck (Abb.9) den Vorsitz der DGfL, den er bis 1978 innehatte. Er studierte Chemie in Heidelberg und Halle, wo er 1939 bei K. Ziegler promovierte und 1943 habilitierte. Ab 1949 war er Professor für organische Chemie und Photochemie

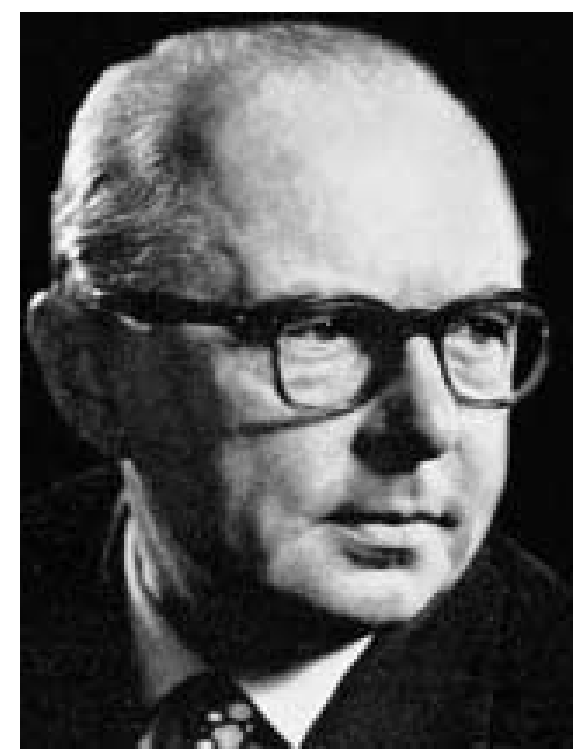

Abb. 7 Prof. Rudolf Schulze, Hamburg, 1906-1974,

Vorsitzender der DGfL 1965- 1974 (Bild aus [23]).

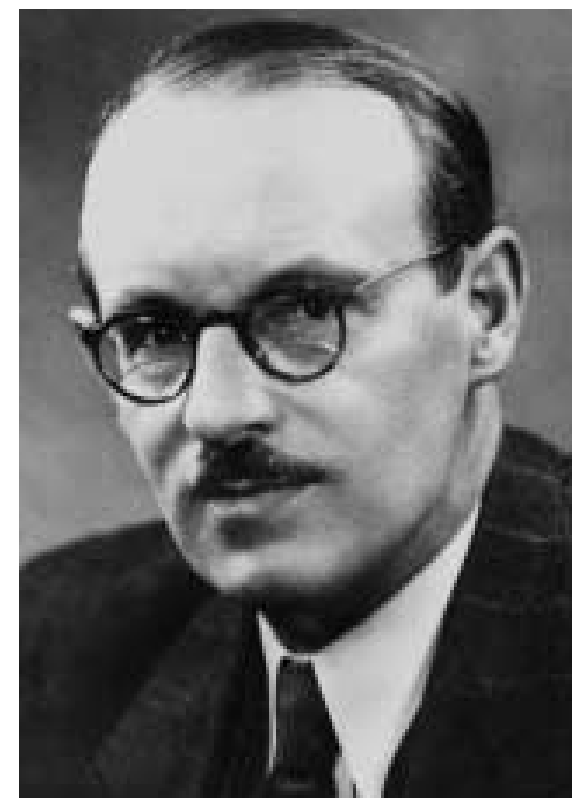

Abb. 8 Dr. Ernst O. Seitz, Hanau, 1901 - 1973, Sekretär der DGfL 1959-1968 (Foto: Familie Seitz).

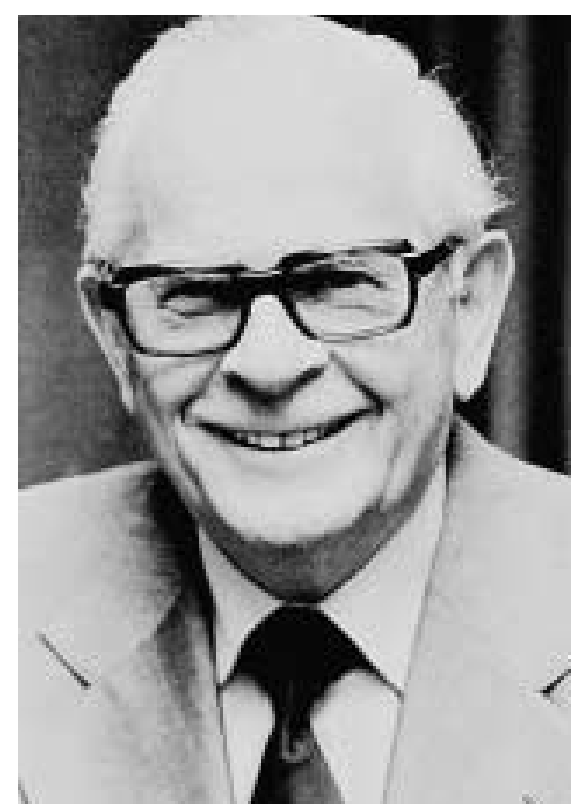

Abb. 9 Prof. Günther O. Schenck, Mülheim/Ruhr, 1913-2003, Vorsitzender der DGfL 1974-1978 (Foto: privat). 


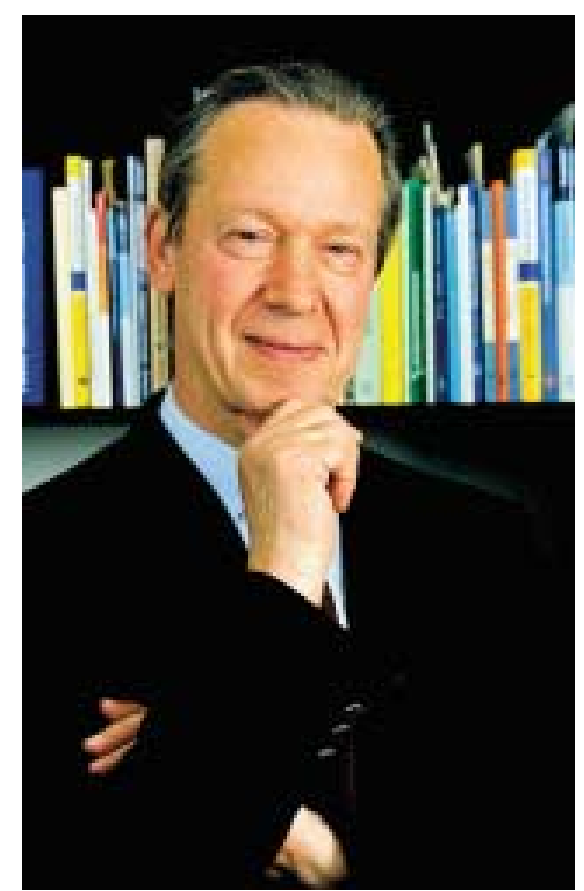

Abb. 10 Prof. Arthur Wiskemann,

Hamburg, geb. 1922, Sekretär der DGfL 1968 - 1976 und Vorsitzender 1978 - 1985 (Foto: privat).

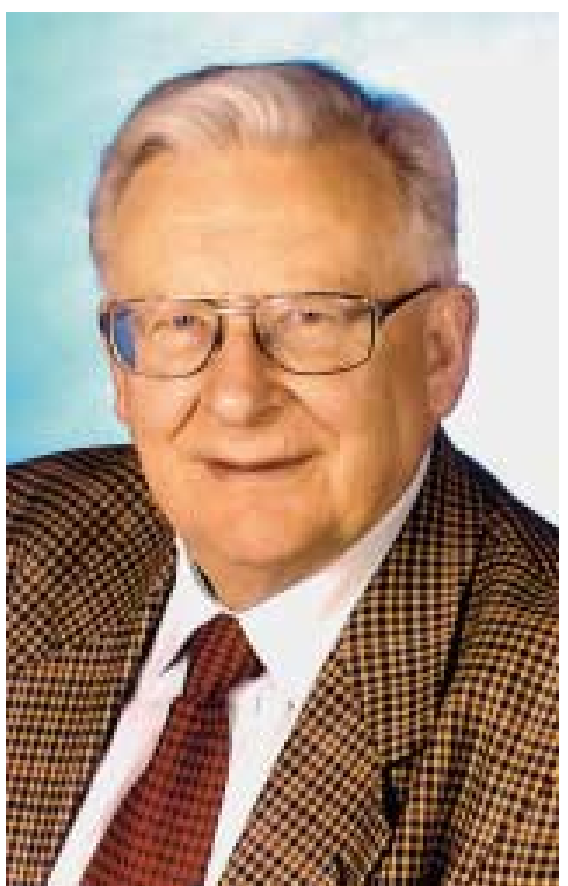

Abb. 11 Dr. Volker Schaefer, Hanau, geb. 1932, Sekretär der DGfL 1976- 1995 (Foto: privat) an der Universität Göttingen, welcher er 1952 - 1953 als Dekan seiner Fakultät besonders diente. Im Jahr 1957 erfolgte die Berufung als Gründungsdirektor des Instituts für Strahlenchemie an das Max-Planck-Institut für Kohlenforschung in Mülheim/Ruhr. Er führte dieses Institut 25 Jahre lang und brachte es zu internationalem Renommee. Am 25.3.2003 verstarb er, kurz vor seinem 90. Geburtstag.

Als Sekretär der DGfL amtierte A. Wiskemann (Abb.10) weiter bis 1976 und wurde abgelöst durch Herrn Dr. Volker Schaefer, Hanau, der das Amt 1976-1995 führte (Abb.11). Das Amt des Kassenwartes ging von Prof. H. Brücker 1972 an Prof. Horst-Dieter Mennigmann über, der dieses bis 1993 versah.

Während die nachfolgenden Jahrestagungen der DGfL weiter Bezüge zu Grundlagenbereichen suchten, 1974 mit den Biologen in Berlin über Strahlungsquellen und Phototropismen, 1976 in Heidelberg mit den Dermatologen über Melaninpigmente, in Konstanz 1977 mit den Photochemikern und 1978 mit den Biophysikern in Ulm, so griff sie das angebrochene Zukunftsthema der Photochemotherapie in einem speziellen, deutsch-schwedischen Symposium „Photomedizin“ am 23. - 25.4.1975 in Oberursel (bei Frankfurt/Main) energisch auf. Es wurde von der DGfL unter ihrem Vorsitzenden Günther O. Schenck zusammen mit der Königlich-Schwedischen Akademie der Ingenieur-Wissenschaften ausgerichtet und in großen Teilen publiziert [25]. Dies brachte den Durchbruch der PUVA-Therapie der Psoriasis in Europa. Eine Fülle von experimentellen und klinischen Arbeiten führten zur Optimierung und Differenzierung dieser Therapie und zur Ausweitung und Festlegung des Indikationsspektrums. Themenbedingt erfolgte eine gewisse „Dermatologielastigkeit“.
Ein neuer Höhepunkt der Lichtforschung bahnt sich seit 1970 an!

Und wieder geht er von der Lichtbehandlung einer häufigen Hautkrankheit, der Psoriasis, aus und entfaltet sich rasch in die angestammten Bereiche der DGfL: Lichtbiologie, Gerätetechnik und spezielle Therapien. Mit der Festlegung des Wirkspektrums bei der Psoriasis in UVA und UVB kommt es zur Reaktivierung der nun „selektierten UV-Therapie der Psoriasis“ (SUP).

Die hochdosierte UVA1-Behandlung der akuten Neurodermitis atopica bringt enormen experimentellen und klinischen Forschungsbedarf und aktualisiert die Frage nach den möglichen Langzeitschäden der verschiedenen Verfahren.

Die zunehmende Zahl von Photoallergien führt neben den klinischen Problemen zu entsprechenden standardisierten Testreihen. Nachdem erreicht wurde, dass bei neuen Medikamenten und Körperpflegemitteln eine prospektive Risikobeurteilung zur photoallergischen Potenz erforderlich ist, und daraus auch Konsequenzen gezogen wurden, konnte eine deutliche Reduzierung der Photoallergien erreicht werden. Ein beachtlicher Erfolg konsequenter Zusammenarbeit, auch im Rahmen der DGfL.

Die Fokussierung erfolgt klar in Richtung klinischer und experimenteller Dermatologie mit engen Bezügen zur zellulären und molekularen Biologie. Dies spiegelt sich auch in der zunehmenden Zahl Dermatologen in der DGfL wider, bei konstanter Zahl von ca. 100 Mitgliedern und in der Übernahme von Vorstandsaufgaben durch Dermatologen, die seit 1978 mit dem Vorsitz betraut sind:

Prof. Dr. Arthur Wiskemann (Abb.10), Direktor der Abteilung Dermatologische Radiologie der Universitätshautklinik Hamburg von 1973-1987, übernimmt von Prof. G. O. Schenck den Vorsitz der DGfL für die Jahre 1978 - 1985. Ihm folgt Prof. Ernst G. Jung, Direktor der Universitätshautklinik Mannheim der Uni- 


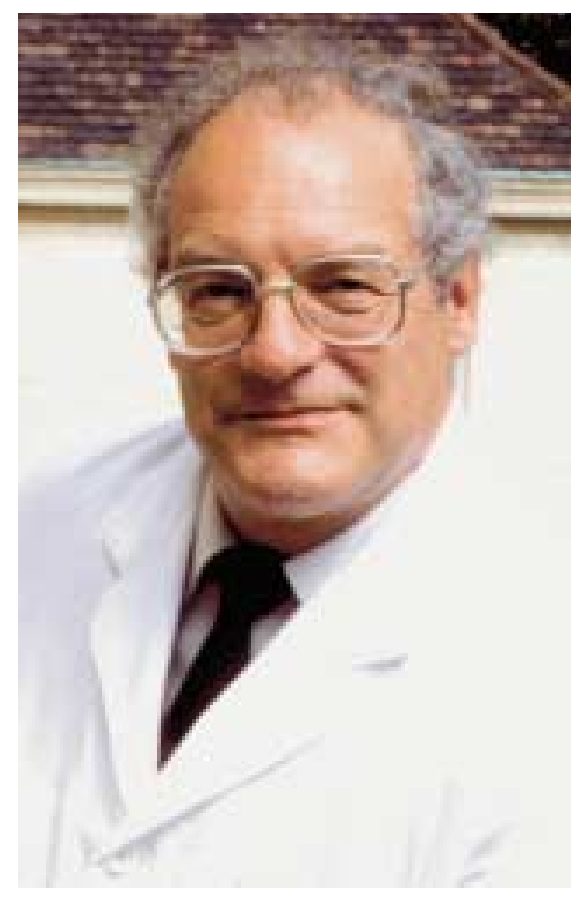

Abb. 12 Prof. Ernst G. Jung, Mannheim, geb. 1932, Vorsitzender der DGfL $1985-1995$ (Foto: privat). versität Heidelberg (1975 - 2000) im Vorsitz der DGfL für die Jahre 1985-1995 [26] (Abb.12). Herr Dr. Volker Schaefer, Hanau, versieht die Geschäfte der DGfL als Sekretär mit zuverlässiger Umsicht und Konstanz von 1976 bis 1995. Die Kassenführung obliegt 1993-1999 Herrn Dr. Peter Bocionek und ab 1999 Herrn Gerhard Saalmann.

Die DGfL tagt seit 1980 in Westerland/Sylt regelmäßig in 3-jährigen, neuerdings in 2-jährigen Abständen zusammen mit der DDG anlässlich deren Tagungen 1982 in Wien und 1985 in Zürich. Dies gelingt auch, als 1987 der 17. Weltkongress für Dermatologie in Berlin durch die DDG abgehalten wird, und setzt sich fort in München 1988 und in Hannover 1990.

Ab 1991 wird konsequenterweise die Zeitschrift „Aktuelle Dermatologie“ des Georg Thieme Verlags, Stuttgart, das offizielle Organ der DGfL und löst die „Strahlentherapie“ als solches ab.

Vom 13.-15. 3. 1992 findet in Düsseldorf wiederum ein spezielles Symposium der DGfL statt, das sich mit der photodynamischen Therapie, der extrakorporalen Photopherese und mit Mechanismen der Photoimmunologie beschäftigt. Damit sind Zeichen neuer Entwicklungen gesetzt.

\section{Neue wissenschaftlichen Organisationen entstehen}

Anlässlich der gemeinsamen Tagung der DDG und der DGfL in Düsseldorf 1993 scheidet die DDG eine Arbeitsgemeinschaft „Photodermatologie“ aus, der u.a. die Prüfung, Qualitätssicherung, und die Evidenzerarbeitung zur Einführung neuer Verfahren obliegt und die Vorbereitung von Stellungsnahmen der DDG. Die AG „Photodermatologie“ und die DGfL arbeiten in der Folge eng zusammen und halten ihre Tagungen gemeinsam ab.

Im Jahr 1994 konstituiert die DGfL zusammen mit der Lichttechnischen Gesellschaft (LiTG) einen gemeinsamen Fachausschuss

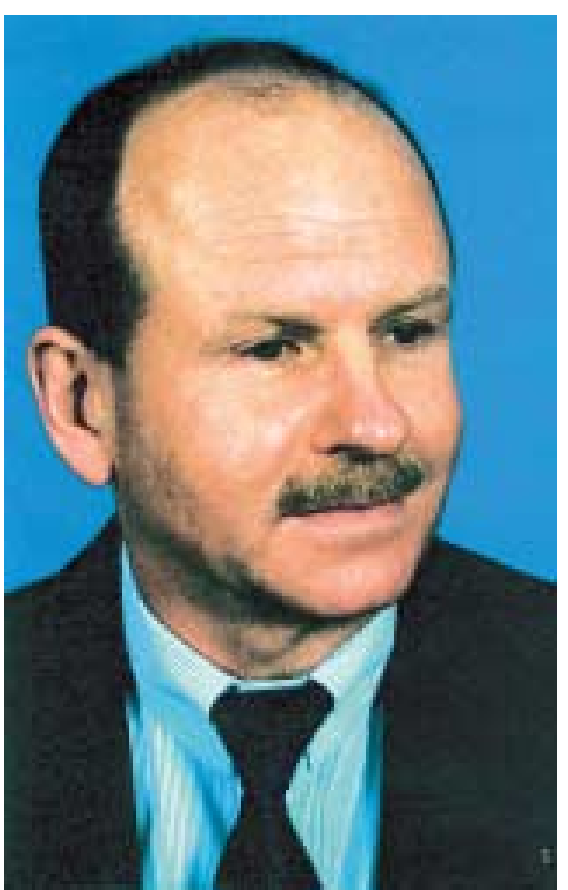

Abb. 13 Prof. Erhard Hölzle, Oldenburg, geb. 1947, Vorsitzender der DGfL seit 1995 (Foto: privat).

„Strahlenkunde“, um den anfallenden technischen Anforderungen schneller und adäquater begegnen zu können.

Während in Deutschland Kooperationen mit gemeinsamen Interessen gebildet werden, kann man im Europäischen Bereich eine Tendenz zur Spezialisierung beobachten. Schon 1986 wurde in Grenoble/F die „European Society of Photobiology“ (ESP) gegründet, die sich alle 2 Jahre an wechselnden Tagungsorten trifft. Und 1998 konstituierte sich in Nizza zudem eine „European Society for Photodermatology“ (ESPD), welche ihre erste Tagung 1999 in Amsterdam abhielt.

\section{Neue Schwerpunkte}

Nach dem Krieg waren, im Rahmen der sprießenden Möglichkeiten, die sportliche Ertüchtigung, die Körperpflege und die Hygiene vordringliche Probleme der Gesundheitserziehung. Diese wurden von Mitgliedern der DGfL im Rahmen der „Kosmetiktage Karlsruhe“ jahrzehntelang mitgestaltet. Probleme des Lichtschutzes und der kosmetischen Bräunung, Handhabung der Lichtexposition in Beruf, beim Sport und in der Freizeit, und die besondere Belastung der Haut bei Fernreisen und Freizeitexzessen nehmen immer mehr zu und werden verantwortlich gemacht für die enorme Zunahme der lichtbedingten Hauttumoren, speziell der Melanome. Zur Belastung durch unkontrollierte Sonnenexposition kommen in vermehrtem Maße auch akute und chronische Überbelastung der Haut durch künstliche Strahlenquellen (Solarien) mit dem Ziel der permanenten Bräunung oder im Rahmen einer übersteigerten „Wellness-Propaganda“ [27]. Die vorzeitige Lichtalterung und die Photokarzinogenese stellen vordringliche Aufgaben dar. Prävention, Früherkennung und Therapie beschäftigen die DGfL sowie die Dermatologie immer mehr.

Im Jahr 1995 erfolgte der Wechsel im Vorsitz der DGfL von Prof. Ernst G. Jung zu Prof. Erhard Hölzle (Abb.13), Direktor der Klinik für Dermatologie und Allergologie in Oldenburg. Dieser wird be- 


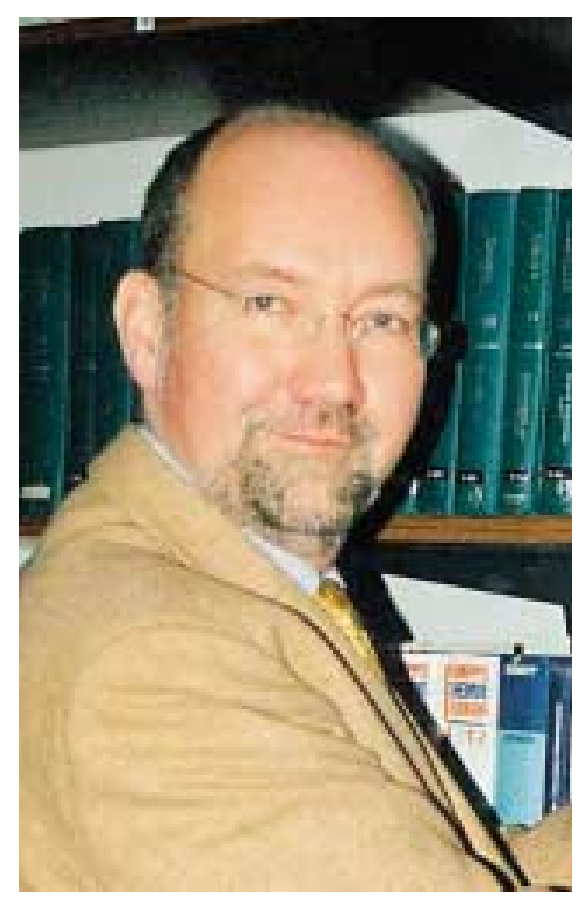

Abb. 14 Prof. Jan Krutmann, Düsseldorf, geb. 1959, Sekretär der DGfL seit 1995 (Foto: privat)

gleitet vom Wechsel des Sekretärs von Dr. Volker Schaefer zu Prof. Dr. Jan Krutmann, Direktor des Instituts für umweltmedizinische Forschung an der Universität Düsseldorf (Abb.14). Sie führen die DGfL wiederum in eine neue und faszinierende Phase der lokalen und systemischen Strahlenwirkung auf den Menschen und ganz besonders dessen Immunapparat. Experimentelle Befunde und zunehmend auch klinische Evidenzen für eine strahlungsinduzierte Modifikation der zellvermittelten Spättypallergie häufen sich. Immunstimulation und Toleranzinduktion beim allergischen Kontaktekzem und bei der Abstoßungsreaktion durch spezielle und gezielte Bestrahlungsverfahren kommen in den Bereich experimenteller Möglichkeiten. Ein neues Kapitel der Photoimmunologie fasziniert seit gut 10 Jahren die Fachleute.

Diese vordringlichen Themen okkupierten die Tagungen der DGfL anlässlich der DDG-Kongresse in Berlin 1995, Karlsruhe 1997, Düsseldorf 2000 und ab 2001 regelmäßig in Berlin. Beachtenswert erscheint, dass experimentelle Befunde und therapeutische Möglichkeiten „Hand in Hand“ gehen und zu gesicherten, evidenzbasierten Aussagen führen. Themenbedingt erfolgte eine gewisse „Dermatologielastigkeit“, welche von der ursprünglichen Intension der DGfL abweicht. Diese hat ausdrücklich die Verbindung und die gegenseitige Befruchtung der verschiedenen Fächer zur Promotion von Lichtforschung und Photobiologie zum Ziel. Dies gilt sicher auch weiterhin und möchte wieder angestrebt werden.

Die DGfL hat an ihrer Jahrestagung vom 7.5.2003 die Namensänderung in „Deutsche Gesellschaft für für Photobiologie“ beschlossen.
Danksagung

Für Hilfen und Informationen danken wir: Prof. F. Bahmer und seinen Kollegen Prof. H. J. Habermalz und Dammann in Bremen, Drs. Vera Enke und W. Knobloch vom Archiv der Berlin-Brandenburgischen Akademie der Wissenschaften in Berlin, Prof. W. Eckart, Institut für Geschichte der Medizin der Universität Heidelberg, den früheren und den gegenwärtigen Mitgliedern des Vorstandes der DGfL.

\section{Literatur}

${ }^{1}$ Lentner A. Geschichte der Lichttherapie. (Dieses Buch wurde 1992 dank der WE-Heräus-Stiftung allen Mitgliedern der DGfL geschenkt.) Photo-Druck Mainz, Aachen: 1992

${ }^{2}$ Levental Z. Der „Sonnendoktor“ Arnold Rikli (1823-1906). Gesnerus 1977; 34: $394-403$

${ }^{3}$ Urbach F, Forbes PD, Davies RE, Berger D. Cutaneous Photobiology: Past, Present and Future. J invest Dermatol 1976; 67: 209-224

${ }^{4}$ Jung EG. Lichtbiologie und Lichttherapie im deutschsprachigen Raum.

Akt Dermatol 1997; 23: 15-18

5 Deutsche Gesellschaft für Lichtforschung und Heliotherapie. Mitteilung Strahlentherapie 1927; 24: 576

${ }^{6}$ Meyer H. Eröffnungsansprache des Vorsitzenden der DGfL. Strahlentherapie 1928; 28: I-IV

${ }^{7}$ Meyer H. Veröffentlichungen der Historischen Kommission für Hessen 1979; 5 : $318-319$

${ }^{8}$ Proppe A. Hans Meyer zum 80. Geburtstag. Hautarzt 1957; 8: $333-335$

${ }^{9}$ Braunbehrens H v. Ein Leben der Röntgenologie. Hans Meyer zum 85. Geburtstag. Med. Klinik 1962; 57: 1300-1302

${ }^{10}$ Scherer E, Habermalz H-J. Prof. Dr. Hans Meyer (1877-1964) Strahlenther Onkol 2002; 178: 295-298

11 Tagungsankündigung der DGfL. Strahlentherapie 1928; 27: 803 und 29:614

12 Bericht der Jahrestagung der DGfL in Münster. Strahlentherapie 1929; 34: 544-698

${ }^{13}$ Bericht der Jahrestagung der DGfL in Dresden 1930. Strahlentherapie 1931; 40: 601 - 794

${ }^{14}$ Steenbeck M. Walter Friedrich, Nachruf. Phys. Bl. 1969; 25: 37 - 38

${ }^{15}$ Schierhorn E. Walter Friedrich Biographien Bd. 69. BSB B. G. Teubner Verlagsgesellschaft, Leipzig: 1983

${ }^{16}$ Dorno C. Meteorologisch-Physikalisches Observatorium Davos. Strahlentherapie 1928; 28: 815-816

17 Vince-Prue D, Hall DO. International Co-operation in Photobiology, a history of C.I.P. Photochem Photobiol 1975; 22: 77-82

${ }^{18}$ Erste Internationale Lichtkonferenz in Lausanne und Leysin im September 1928. Mitteilung Strahlentherapie, 1928; 27: 802

${ }^{19}$ Schreiber H (Hrsg). Kongressbericht des 3. Internationalen Kongress für Lichtforschung, 1. - 7.9.1936 in Wiesbaden. DGfL 1938. Von 98 Vorträgen sind 42 erschienen in Strahlentherapie 1938; 61 :403 - 715,

${ }^{20}$ Seitz EO. Jahrestagung der DGfL am 26.11.1959 in Bad Nauheim. Strahlentherapie 1960; 111: 487

${ }^{21}$ Pohlit W. Boris Rajewsky zum 70. Geburtstag am 19. Juli 1963. Strahlentherapie 1963; 121: 21 - 22

22 Becker J, Kuttig H. Boris Rajewsky 1893 - 1974, Nachruf. Strahlentherapie 1975; 149: $532-533$

23 Schenck GO. Prof. Rudolf Schulze, 1906 - 1974, Nachruf. Strahlentherapie 1975; 150: 227-233

24 Schenck GO (Hrsg). Progress in Photobiology. Proceedings of the 7. International Congress on Photobiology 21.-25.8.1972 in Bochum. Library of Congress USA 1973, Cat. Nr. 73-85281,

${ }_{25}$ Jung EG (Hrsg). Photochemotherapie; Grundlagen, Technik und Nebenwirkungen. Stuttgart: Schattauer, 1976

${ }^{26}$ Jung EG. Die Historie: Anfänge und Entwicklungen der Dermatologie in Mannheim. Akt. Dermatol. 2001; 27: 401 - 408

27 Jung EG. Sonnenkultur und Sonnenkult. Ärztl. Kosmetologie 1969; 19: $114-116$ 